\title{
GJ 436c? The contribution of transit timings
}

\author{
B.-O. Demory ${ }^{1}$, M. Gillon ${ }^{1}$, C. Waelkens ${ }^{2}$, D. Queloz ${ }^{1}$ and S. Udry ${ }^{1}$ \\ ${ }^{1}$ Observatoire de l'Université de Genève, Switzerland \\ email: brice-olivier.demory@obs.unige.ch \\ ${ }^{2}$ Instituut voor Sterrenkunde, KU Leuven, Belgium
}

\begin{abstract}
From recent high-accuracy transit timings measurements, we discard the $5 M_{\oplus}$ planet recently proposed by Ribas et al. (2008). Thanks to a combined radial-velocity and transit timings overview we also define a mass/period domain in which a secondary planet may be found in the system. We also show that timings obtained until now, although not sufficient to remove degeneracies on mass and period, can still restrict the parameter space of the potential secondary planet.
\end{abstract}

\section{Introduction}

Discovered by Butler et al. (2004), the Neptune-sized planet orbiting GJ 436 has trig- gered a great deal of observational efforts. Its transiting nature observed by Gillon et al. (2007b) gave the opportunity to constrain its internal structure (e.g. Adams et al. 2008) and provided hints about the composition of its atmosphere. The observed significant eccentricity naturally lead several authors to invoke the existence of a potential additional planet. Space and ground-based observations obtained from 2004 to 2007 and publicly available provided a better understanding of the stellar activity and showed no significant hint about another planet in the system (Demory et al., 2007) as well as a detailed explanation regarding the b planet eccentricity and circularization time (Deming et al., 2007). Recently, Ribas et al. (2008) have reported the possible discovery of a $4.8 M_{\oplus}$ planet orbiting GJ 436 in an outer 2:1 mean motion resonance $(5.2 \mathrm{~d})$ based on radial-velocity dataset from Maness et al. (2007). From high-accuracy transit timings obtained by Spitzer, with the camera on Euler Swiss telescope and more recently by the Mercator belgian telescope and the TCS (Alonso et al. 2008), we explore here a preliminary contribution of transit timings regarding an additional planet in GJ 436 system.

\section{A $4.8 M_{\oplus}$ planet on a $5.2 \mathrm{~d}$ orbit?}

The starting point of the paper by Ribas et al. (2008) is to invoke an orbital inclination change of GJ 436b that would be induced by an additional companion in the system. They mention a peak appearing in the residuals periodogram of the radial-velocity single planet fit published by Maness et al. (2007). By adding a planet corresponding to the peaks period of $5.18 \mathrm{~d}$, they marginally reduce the global $\chi^{2}$ and note that their proposed planet would be easily detectable by transit timings. The Achilles heel of their assumption is to fit keplerian orbits. Laughlin \& Chambers (2001) clearly show that the configuration proposed by Ribas et al. (2008) cannot be dealt without considering planet-planet interactions instead of simple keplerian fits. We used the Mercury integrator package Chambers (1999) to compute transit timings of GJ 436b considering both direct planet-planet interactions and indirect effects caused by the wobble of the star induced by the potential, secondary planet. By setting initial conditions to the ones of 
Gillon et al. (2007a) for GJ 436b and those provided by Ribas et al. (2008) for the proposed c planet. it appears that the first confirmed transit of GJ 436b would have been more than 15 hours late with regard to the actually observed one. Indeed, perturbations from their planet on the $b$ induce a long-term drift coupled to a short-term oscillation of the transit timings interval of the b planet. Figure 1 reproduces the observed minus calculated epoch assuming perturbations from the proposed planet. We cannot match an $\mathrm{O}-\mathrm{C}=0$ behaviour, even by parsing the full domain covered by error bars published by the authors. Furthermore, we did not manage to match the expected TTV (Transit Timing Variations, cf. Fig. 2) pattern with observations. Such configuration in mean motion resonance is known to show the largest TTV amplitudes. Perturbations from this potential planet would have modulated the period of GJ $436 \mathrm{~b}$ sufficiently to alter the observed transit epochs, in consequence the planet proposed by Ribas et al. (2008) is inconsistent with observations.

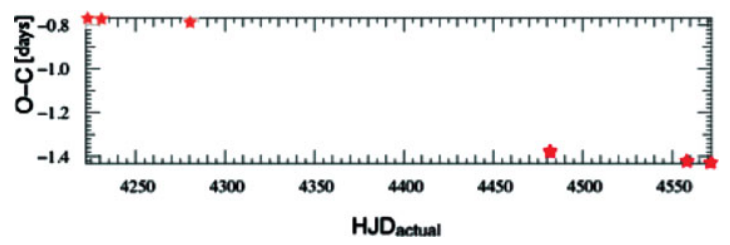

Figure 1. This plot shows the difference in days between expected and actual transit epochs, assuming the existence of the proposed GJ 436c of Ribas et al. (2008). It appears that the first confirmed transit of GJ 436b would have occured 15 hours later than actually observed.

\section{Is any secondary planet supported by current transit timings?}

At this time, we unfortunately do not have sufficient transit timings to lift mass/period degeneracies and a fortiori to conclude about orbital properties of a perturbator. However, going one step further, available timings and especially the most recent transit timing of Mercator allow to constrain a basic mass-period domain in which a planet might exist. This is developed on Fig. 3 where we plot detection limits imposed by radial-velocity observations and the region complying with current maximum transit timing variation (TTV, time interval between successive transits) of 60s estimated from our dataset. For the latter, we used the analytical approach by Holman \& Murray (2005). The black area encompasses all mass/period pairs for which the maximum TTV amplitude induced by the corresponding exterior companion $(\mathrm{e}=0.3)$ on the b planet would be below the maximum estimated TTV amplitude. Furthermore, a planet located in the white area would have induced a TTV signal amplitude above $3 \mathrm{~min}$, the approximative expected TTV for an Earth-mass planet in mean motion resonance with the b. It is crucial to mention that this result is only relevant considering present transit timings since we cannot secure the fact that the current observed maximum amplitude is the actual one. This preliminary TTV analysis shows how the mass/period domain for a potential secondary planet in GJ 436 system may be constrained. It is interesting to note that a dark area for radial velocities is brought to light by TTV. However, without the contribution of radial velocities, a perturbator located in this region would require many more high accuracy timings to characterize its orbital parameters. Once combined with radial-velocity (RV) detection thresholds, it appears that there is still room for a secondary planet located in an area where RV methods would be unable to detect a companion: a 1 Earth-Mass planet at 9 days for instance. 


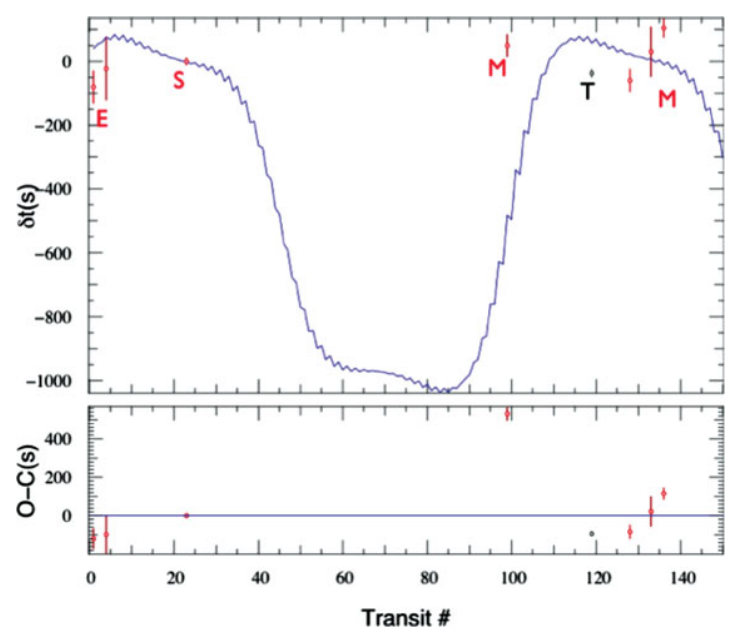

Figure 2. Expected TTV of GJ 436b considering perturbations from the secondary planet proposed by Ribas et al. Since TTV is a relative approach, the model curve (blue) is arbitrarily set at 0 for the Spitzer timing. A period of $2.64390 \mathrm{~d}$ for the $\mathrm{b}$ planet is assumed. This curve is among the best fit to the dataset found so far, considering the large error bars ( $45 \mathrm{~d}$ on $\omega$ ) provided by Ribas et al. (2008). However, this model is highly inconsistent with first Mercator and TCS data points. Timings sources : E stands for Euler, S for Spitzer, M for Mercator and $\mathrm{T}$ for TCS.

\section{Bibliography}

Adams, E. R., Seager, S., Elkins-Tanton, L. 2008, ApJ, 673, 1160

Alonso, R., Barbieri, M., Rabus, M., Deeg, H. J., Belmonte, J. A., Almenara, J. M. 2008, ArXiv preprints

Butler, R. P., Vogt, S. S., Marcy, G. W., Fischer, D. A., Wright, J. T., Henry, G. W., Laughlin, G., Lissauer, J. J. 2004, ApJ, 617, 580

Chambers, J. E. 1999, MNRAS, 304, 793

Demory, B.-O., et al. 2007, A\&A, 475, 1125

Deming, D., Harrington, J., Laughlin, G., Seager, S., Navarro, S. B., Bowman, W. C., Horning, K. 2007, ApJ, 667, L199

Fortney, J. J., Marley, M. S., Barnes, J. W. 2007, ApJ, 659, 1661

Gillon, M., Pont, F., Demory, B.-O. et al. 2007, A\&A, 472, L13

Gillon, M., Demory, B.-O., Barman, T, et al. 2007, A\&A, 471, L51

Holman, M. J., Murray, N. W. 2005, Science, 307, 1288

Laughlin, G., Chambers, J. E. 2001, ApJ, 551, L109

Maness, H. L., Marcy, G. W., Ford, E. B., Hauschildt, P. H., Shreve, A. T., Basri, G. B., Butler, R. P., Vogt, S. S. 2007, PASP, 119, 90

Ribas, I., Font-Ribera, A., Beaulieu, J.-P. 2008, ApJ, 677, L59 


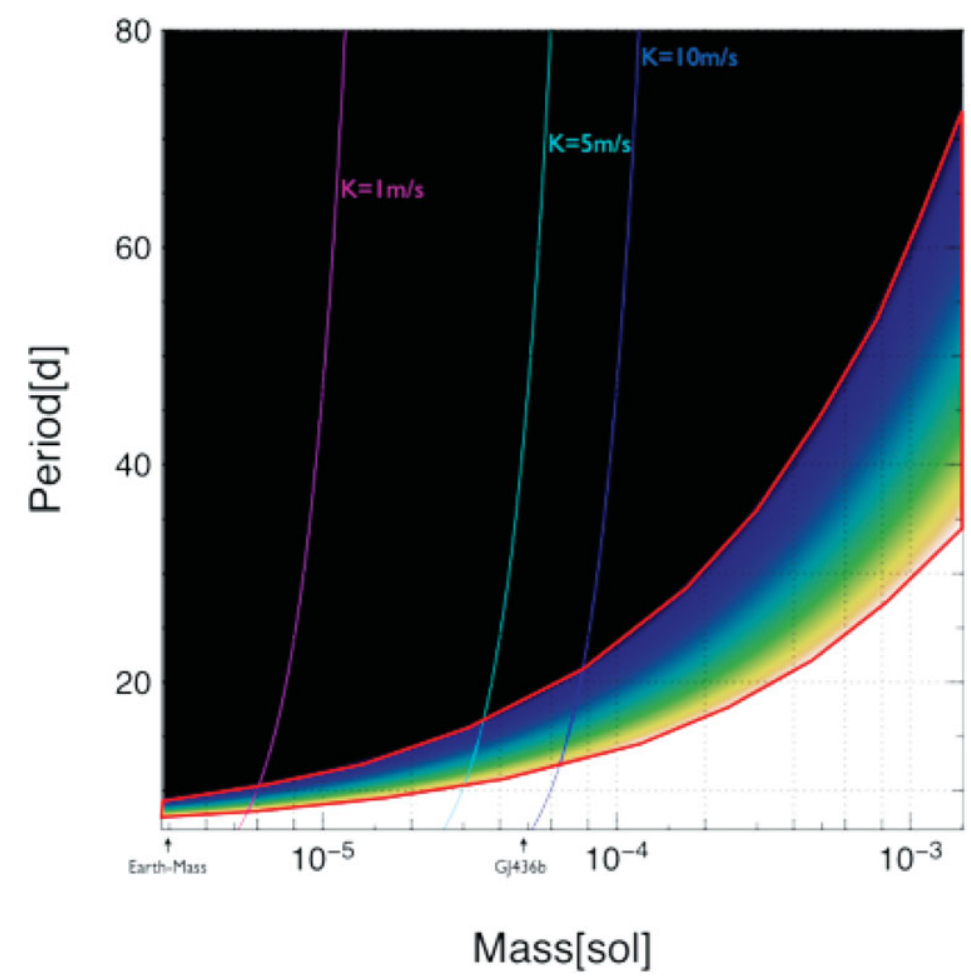

Figure 3. This figure shows the region (red) complying with current maximum transit timing variation (TTV, time interval between successive transits) of 60 s estimated from our dataset on GJ 436b. Both black and white areas are not compatible with observed timings. We used the analytical approach by Holman \& Murray (2005) to illustrate the area (in black) that encompasses all mass/period pairs for which the maximum TTV amplitude induced by an outer planet $(\mathrm{e}=0.3$ ) would be below the maximum observed TTV amplitude. Furthermore, a planet located in the white area would have induced a TTV signal amplitude above 3 min, the approximative expected TTV for an Earth-mass planet in mean motion resonance with GJ 436b. Coloured gradient represents TTV amplitude, from 60s (blue) to 3min (white). Magenta, cyan and blue curves show the RV signal amplitude corresponding to 1,5 and $10 \mathrm{~m} / \mathrm{s}$ respectively. 